At aphelion in this orbit the comet would be distant from the orbit of Jupiter $0^{\circ} 50_{3}$, but there is a very much closer approach to the orbit of Mars, at a true anomaly of $37^{\circ} \mathbf{1} 3^{\prime}$, corresponding to heliocentric longitude $343^{\circ} 25^{\prime}$, where the distance of the two orbits is only 0.0088 , and it is worthy of note that between April 5 and 10, I868, both Mars and the comet would pass that point, and if Dr. Berberich's period is approximately correct, there must have been a close approach of the two bodies, possibly a closer one than calculation assigns. The following positions are deduced from the elliptical orbit:-

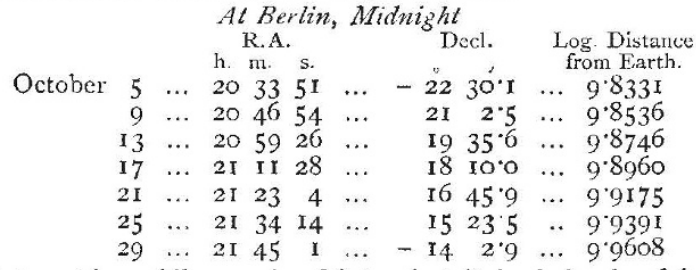

The comet is rapidly growing fainter, but it is obviously of importance for its theory that observations should be continued as long as possible.

COMET I 884 c. - A new comet was discovered by Herr Wolf at Heidelberg on September I7, and was observed at Strasburg on September 20. It was also independently detected by Dr. Copeland at Dun Echt on September 22, the night before the telegraphic notice from Kicl arrived at that Observatory. Prof. Tacchini has favoured us with the following observations made by himself and Prof. Millosevich at the Observatory of the Collegio Romano in Rome:-

$$
\text { Rome M.T. R.A. Decl. }
$$

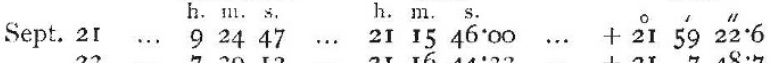

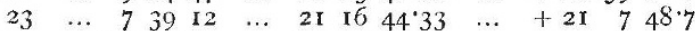

This comet is likely to remain visible for several months, according to the orbits yct calculated, but a somewhat wider extent of observation than is now available will be required to predict its track in the heavens with any degree of certainty.

THE Lunar Eclipse on OCtober 4.-We gave last week the times of occultations of two stars during the totality of this eclipse, of which accurate positions are found in our catalogues. A somewhat extensive list of stars liable to occultation has been determined at Pulkowa with sufficient precision for the predictions of the times of immersion and emersion, which have been communicated to various observatories. Several stars rated higher than the ninth magnitude appear on this list, where the Durchmuster ung magnitudes are followed, Our remark last week should have been explained as referring only to stars of which accurate places are found in the catalogues.

\section{HYDROXYIAMINE AND THE ASSIMILATION OF NITROGEN BY PLANTS}

THE researches of $V$. Meyer and $E$. Schulze on the action of hydroxylamine salts upon plants (Berl. Ber., xvii. 1554) were undertaken with the a priori probability of showing that this base plays an important part in the synthetical activity of the plant ; and although they have not succeeded in establishing the experimental fact, the results obtained are of great interest, and the whole is emincntly suggestive of future possibilities. The supply of nitrogen to plants takes the form of nitrates and ammoniacal salts, and these classes of compounds being destitute of synthetical activity, we are driven to assume that the earlier stages of nitrogen assimilation consist in the conversion of these comparatively inert substances into derivatives having the "chemical tension" necessary to synthetic activity. Hydroxylamine and its salts, which occupy in point of oxidation a position intermediate between ammonia and the nitrates, are bodies possessing this character in the highest degree. To use the author's words, "the behaviour of this base towards the organic oxy-compounds is most aggressive : it is indeed astonishing with what facility it converts carbonyl-compounds into azotised derivatives." This is notably the case with the ketones and aldehydes of the fatty series, the products of the union being oximidoderivatives, e.g. aldoxime, acetoxime, isonitroso acids; in these the characteristic $\mathrm{C}=\mathrm{NO}_{2}$ group easily undergoes reduction, with formation of the corresponding amido derivatives; and upon the hypothesis of the formation of hydroxylamine in the plant as the first stage in nitrogen assimilation, it is easy to see in what manner its non-nitrogenous constituents, which for the most part possess the characteristics of aldehydes and ketones, would contri'sute to the further stages in its elaboration.

To test this hypothesis, in the first instance, the authors instituted parallel experiments on the culture of maize, to which the nitrogen was supplied in the form of ammonium sulphate, hydroxylamine sulphate, and hydrochlorate and potassium nitrate, respectively. The result was, in a word, to show that the hydroxylamine salts act as direct poisons to plant life, as indeed they have already been shown by Bertoni to act towards animal life. Having established this fact, the authors inferred their probable action a i antiseptics, and experiment showed that they possess this property in a remarkable degree. This result, as they contend, does not negative the original hypothesis, as the occurrence of the base in the plant would necessarily be transitional.

It seems to us that the antiseptic properties of hydroxylamine are a dire t consequence of its synthetical activity; and further that antiseptics fall into three classes according to their disturbance of one of the three essential conditions of cell life, which are : (I) hydration; (2) oxidation; (3) the synthetical activity of aldehydes (Löw and Bokorny), chiefly in the direction of condensation. In illustration of this classification we may cite as typical members of group (I) common salt, (2) sulphurous acid in its various combinations, (3) phenols.

\section{THE BRITISH ASSOCIATION REPORTS}

Second Report of the Committee, consisting of Prof. 4.W. Williamson, Chairman, Profs. Sir H. E. Roscoe, Dewar, Frankland, Crum-Brozen, Odling, and Armstrong, Messrs. A. VernonHarcourt, J. Millar Thomson, V. H. Veley, F. Japp, H. Forster Minley, and H. B. Dixon (S.cretary), appointed for the purpose of drawing up a Statement of the Varieties of "Chemical Numes" which have come into use, for indicating the causes which have led to their adoption, and for considering what can be done to bring about some Convergence of the Viezes on Chemical Nomenclature oblaining amons Enolish and Foreign Chemists.- The Report is somewhat lengthy, and includes some long tables of varietics of names for common chemical substances. It commences with historical notes on chemical nomenclaturc. No attempt was masle until about 100 years ago to name chemical substances in a way which would indicate their composition; alchemistic or " culinary" names being given to substances in many cases. Macquer is credited with being the first to introduce generic names like vitriol and nitre to indicate sulphates and nitrates. The term salt was used to indicate almost any substance soluble in water and affecting the sense of taste, and in the eighteenth century acids, salts, and bases began to be distinguished. Rouelle was the first to define a salt from its chemical properties, and distinguish it from acids and bases (see Kopp's "History of Chemistry," iii.). Bergmann and Guyton de Mouveau separately proposed systems of nomenclature, many terms of which are still in use. De Mouveau made the terminations of names of acids uniform, and the names of salts to indicate their composition from bases and acids. In 1787 Lavoisier, De Mouveau, Berthollet, and Fourcroy prepared a scheme of naming compounds which is practically that in common use now, introducing the terminations "ate" and "ic," "ite" and "ous," in acids and salts. But higher and lower oxide; are not distinguished by generic namcs. Berzelius made a more elaborate classification of salts, and added some names. He distinguished the halogen compounds of bydrogen as hydracids, and distingtished clearly between "neutral," "acid," and "basic" salts. The views now held of acids, salts, and bases are practically those of Gerhardt and Laurent, who first recognised the part pla! ed by hydrogen in acids and salts. The Report then goes on to consider the tables, which give the number of times a substance has been distinguished by any particular name. Table I. deals with the names of oxides of carbon from $\mathbf{7} 755$ to I882. By far the greater number of sources give the names carbonic oxide to $\mathrm{CO}$ and carbonic acid to $\mathrm{CO}_{2}$; systematic names like carbonous oxide and carbonic oxide only occurring two or three times, the terms carbonic oxide and carbonic anhydride or dioxide being next in frequency. In France and Germany the names oxide de carbon and acide carbonique, Kohlenoxyd and Kohlensäure have been much more frequently used. But in several instances the same names have been used in a different sense; the term carbonic oxide being some- 